\title{
Essential prescribing tips for GP Associates-in-Training
}

Mrs Gill Gookey

Medicines Management Team, NHS Rushcliffe CCG, Nottingham

Email: gillian.gookey@nhs.net

Dr Richard Knox

School of Medicine, University of Nottingham, Medical School, QMC, Nottingham

Dr Nde-Eshimuni Salema

Division of Primary Care, School of Medicine, University of Nottingham, Nottingham

Ms Kate Marsden

Division of Primary Care, School of Medicine, University of Nottingham, Nottingham

Dr Brian Bell

Division of Primary Care, School of Medicine, University of Nottingham, Nottingham

Mrs Mindy Bassi

Nottingham City CCG, Nottingham

Dr Nick Silcock

Wollaton Park Medical Centre, Wollaton Park, Nottingham

Mr Glen Swanwick

Division of Primary Care, School of Medicine, University of Nottingham, Nottingham

Professor Anthony Avery

School of Medicine, University of Nottingham, Medical School, QMC, Nottingham

Prescribing is an essential role in general practice but it is also, at times, a high risk activity.

GP Associates-in-Training (GP AiTs) have been highlighted as needing further support to reduce the risk of prescribing errors. This article highlights some common prescribing errors to help GP AiTs to review their prescribing and develop prescribing habits to avoid errors. The general practice workforce is changing and there are more pharmacists working in general practice. This article describes the role of clinical pharmacists in prescribing safety and in supporting GP AiTs. 


\section{The GP curriculum and essential prescribing tips for GP Associates-in-Training}

Contextual statement 2.04: Enhancing professional knowledge requires GPs to be able to apply evidence in the context of the patient, the community and the healthcare setting, specifically to:

- Provide effective and evidence-based prescribing, adhering to the GMC's principles of good medical prescribing.

- Use your knowledge of the literature and evidence to solve and manage clinical problems.

- Apply the findings from research, national guidance and audit in the context of dayto-day clinical and organisational management of patients.

- Realise that you will always work better in a multidisciplinary team and that your patients will benefit if the whole team works well; developing skills so that you and your organisation learn together will benefit patient care.

\section{Are prescribing errors an issue for GP AiTs?}

Prescribing errors in both primary and secondary care have the potential to cause significant morbidity and mortality. The GMC PRACtICe study identified prescribing errors in around one in twenty prescriptions in primary care (Avery et al., 2012). The study highlighted that, as a group, GP AiTs may benefit from help to reduce their prescribing errors. 'Based on discussions we have had with GP AiTs, many report a lack of confidence in their role as prescribers and admit to finding the transition from hospital based prescribing to community based prescribing a significant challenge.

All prescribers develop prescribing habits that may reduce or increase the risk of prescribing error. Reflecting on these habits can result in changes that reduce the risk of prescribing error in the long term. A core role for a pharmacist is the identification and prevention of prescribing errors. When a pharmacist is based within GP practice they are ideally placed to focus on prescribing safety. 


\section{Clinical pharmacists in GP practice}

Pharmacists have been working in general practice for a few years in a variety of ways including being directly employed by local NHS organisations such as Clinical Commissioning Groups or directly by GPs. Many pharmacists are now qualified as independent prescribers.

The NHS Five Year Forward View (NHS England, 2014) acknowledged the increasing pressure in primary care, proposed a sustainable strategy for the NHS and suggested a "far greater use of pharmacists". The General Practice Forward View (NHS England, 2016) followed on from this and acknowledged the need to expand the workforce in general practice to meet the increasing demands and included the incorporation of clinical pharmacists into the practice teams highlighting that pharmacists "remain one of the most underutilised professional resources in the system and we must bring their considerable skills in to play more fully". The NHS Clinical Pharmacists in General Practice programme has allowed an increasing number of clinical pharmacists to develop new roles within general practice and has supported pharmacists in obtaining an independent prescribing qualification if necessary. The Next steps on the Five Year Forward View (NHS England, 2017) continues the commitment increase the use of pharmacists in general practice to free up GP time and "ensure efficient use of medicines".

Historically, the pharmacist's role in GP Practice focussed almost entirely on managing prescribing costs and implementing local guidelines but it has now developed to use the pharmacist's skills in a more clinically orientated, patient-centred role with a key focus on medicines optimisation and prescribing safety. The support for the practice based pharmacist outlined in the documents above will ensure that the role continues to develop. 


\section{Pharmacists supporting prescribing safety with GP AiTs}

There are many examples in GP practice of practice based pharmacists providing support and training to GP AiTs either during the induction process or as on-going support during their training. One example is a pilot study in the East Midlands (the 100 prescription study); an educational intervention which involved a clinical pharmacist retrospectively reviewing 100 prescription items issued by each GP AiT and then providing feedback. From this we have created ten 'top tips' that we believe can help support GP AiTs in undertaking a similar review of their prescribing. Where there is a clinical pharmacist available in practice it may be possible to obtain their support in the review. 


\section{Top tips for GP AiTs to consider when reviewing prescribing}

Our work reviewing prescribing errors made by GP AiTs has identified ten themed areas that may help other GP AiTs review and improve their prescribing practice. The ten themed areas are presented with examples seen in real-life practice.

\section{Right guidance.}

Local guidelines and formularies are easy to refer to; they suggest products and dosages specific to the locality and are usually readily accessible on-line. Local guidelines usually reflect any available national guidance e.g. the National Institute for Health and Care Excellence (NICE) or the Scottish Intercollegiate Guidelines Network (SIGN). Whenever possible, prescribing should be in line with up to date local guidance. If this is not possible, the reason should ideally be documented in the clinical record.

Particular consideration should be given to the local antimicrobial guidelines and the local asthma and chronic obstructive pulmonary disease (COPD) guidelines. Antimicrobial guidelines can change relatively frequently and will be specific to the local area with regards to product choice, dosage and course length as they will reflect local antimicrobial sensitivities. Following local antimicrobial guidelines is important to ensure effective antimicrobial stewardship as outlined in NICE guidance (NICE, 2015a). Asthma and COPD guidelines will reflect national guidance but also include the locally preferred product choices and corresponding dosages for each stage in treatment.

Examples of errors include:

Stepping up to $\mathrm{Clenil}^{\circledR} 200$ (two doses twice a day) from salbutamol alone in asthma (local guidelines recommend $\mathrm{Clenil}^{\circledR} 100$, two doses twice a day). 
$>$ Clenil $^{\circledR}$ started first line for a patient with suspected chronic obstructive pulmonary disease (COPD).

$>$ Prescribing doxycycline and amoxicillin together to treat an exacerbation of COPD. Local guidelines recommend one or the other not both together.

$>$ Prescribing a 14 day course of ciprofloxacin for acute prostatitis when the local guidelines recommend 28 days.

\section{Right dose.}

There are numerous reasons why an incorrect dosage might be prescribed. Many guidelines are available in general practice and it is a challenge for prescribers to be aware of all the updates and changes to medication and dosages. Extra care is needed when prescribing a medication that has multiple indications requiring different doses. Dose conversions, especially for opioids are particularly complicated and sometimes confusing. If there is any doubt, the calculation should be double checked with a colleague. Paediatric dosing of medication is another area that is prone to dose errors. Dosing is often based on weight and/or age. The BNF for Children should be referred to and when needed, dosing should be based on an up to date weight and age (BMJ Group \& Pharmaceutical Press, 2017a).

Examples of errors include:

Aciclovir prescribed at a dose to treat cold sores when the patient had shingles.

$>$ Conversion of codeine to morphine in pregnancy that would result in an unintended dose increase.

$>$ Amoxicillin prescribed at a dose of $125 \mathrm{mg}$ for a child of 15 month old child with otitis media when the local guidelines and BNF for Children recommend a dose of $250 \mathrm{mg}$.

$>$ Citalopram dose increased from $20 \mathrm{mg}$ to $30 \mathrm{mg}$ daily in a 74 year old patient by prescribing an additional 10mg tablet. The patient had ischemic heart disease. In December 2011 an MHRA update limited the dose of citalopram to $20 \mathrm{mg}$ per day in patients over 65 years old due to the risk of dose-dependent QT interval prolongation (MHRA, 2011). 


\section{Right formulation and strength.}

Different formulations can change the potency of the medication and there may be more strength options available than realised. Selecting the right formulation and strength of a medication can be further complicated by the introduction of new products to the market. When prescribing electronically the selection lists can be confusing and contain unfamiliar products which may result in the incorrect formulation or strength being unknowingly selected. Nationally there have been incidents (sometimes fatal) due to formulations of high dose opioid liquids being selected instead of the lower strength e.g. morphine $100 \mathrm{mg}$ in $5 \mathrm{ml}$ solution prescribed instead of morphine $10 \mathrm{mg}$ in $5 \mathrm{ml}$ solution.

Examples of errors include:

Hydrocortisone butyrate $0.1 \%$ cream is a potent steroid and was prescribed when standard hydrocortisone cream was intended.

$>$ Diclofenac 3\% gel prescribed for topical pain relief. This strength is licenced for actinic keratosis rather than as a topical anti-inflammatory.

\section{Right combination.}

Consideration should be given to other medication that has been recently prescribed (in primary or secondary care) or purchased to avoid patients taking two similar medications at the same time. The prescribing of brands and branded generics has increased so extra care is needed to ensure a brand is not prescribed as well as a generic.

Examples of errors include:

$>$ Tramadol prescribed with codeine or morphine.

$>$ Two paracetamol products prescribed without clear dosage instructions to avoid the patient taking both. 
Salbutamol issued as an acute on the same prescription on which Ventolin ${ }^{\circledR}$ was issued from repeat.

\section{Right product licence.}

A licenced product should be prescribed if there is a suitable product available. Information on product licences for medication can be found at www.medicines.org.uk. The prescriber should be aware when they are prescribing a product outside of its licence. Prescribing advice on some frequently used unlicensed indications can be found in the BNF (BMJ Group \& Pharmaceutical Press, 2017b).

Examples of errors include:

Ketoconazole shampoo prescribed first line in a 1 year old with cradle cap. Ketoconazole has a licence in adolescents and adults only.

Diclofenac tablets prescribed to a patient with a cardiac history.

Ibuprofen gel recommended to be applied to impetigo on the face. Ibuprofen gel should not be used on broken skin.

\section{Right directions.}

Patients frequently do not remember instructions given verbally in a consultation (Kessels, 2003). Having clear directions on a prescription helps to ensure that medication is used correctly and treatment is optimised. This is particularly important in patients who may have more than one carer or who are prescribed multiple medications. The directions on a prescription will be added to the product label during the dispensing process. The pharmacy will also transfer the same information to the medication administration charts used in care homes. 
Examples of errors include:

$>$ Glaucoma drops prescribed to a care home patient without the treatment eye stated.

$>$ Prescribing morphine sulphate solution 'when required' without stating a frequency or a maximum dosage.

> The directions "Take as directed" for colecalciferol 20,000unit capsules. There is potential for overdose with high strength vitamin $D$ and a clear dosage should always be added to the medication.

> Tramadol 50mg capsules prescribed with the directions "take one or two as needed" with no indication as to the frequency of dosage or the maximum number of capsules that may be taken in a day.

\section{Right review of medication.}

When a patient requests an item that has been previously prescribed, or an annual medication review is undertaken, the suitability of all medication for continued prescribing should be assessed. Starting with whether there is still a valid clinical indication for the medication the prescriber should also assess whether the medication is an appropriate treatment choice for the indication (bearing in mind any changes in guidelines, other current medication and comorbidities of the patient). The current dosage should be re-assessed. Whilst the dose prescribed initially may have been correct, a patient factor could have changed which would require a change to the dosage e.g. change to renal function, weight, or age. It is important to also consider any omissions to therapy e.g. gastroprotection with a non-steroidal antiinflammatory drug in an older patient and whether the patient is purchasing or obtaining medication other than from the practice e.g. from the hospital or pharmacy. With the move towards encouraging patients to self-care for minor ailments and conditions it is even more important to assess what medication is being obtained from other sources than the practice.

Examples of errors include: 
> Prescribing a further supply of hormone replacement therapy (HRT) to a 60 year old patient, whose last medication review was over one year ago, without documenting a discussion regarding risks or considering stopping treatment.

$>$ A medication review coded in a patient prescribed aspirin alone post-stroke. National (Royal College of Physicians, 2016) and local guidelines would suggest clopidogrel instead of aspirin alone or aspirin and dipyridamole in combination.

$>$ Medication review in a patient with cardiac risk who had never been prescribed a statin and had no documented reason as to why it was not prescribed. A review of the clinical notes showed this had been identified a few years previously by a nurse as something that should be discussed at the next appointment with a GP but there was no documentation to say that this had occurred.

$>$ Clotrimazole $1 \%$ cream prescribed with the directions that it should be used in the groin area when the consultation notes state that the rash was under the breasts. The cream was issued as a repeat supply of a previously issued acute prescription but the directions had not been updated.

\section{Adherence to medication}

Adherence to long term medication is known to be a problem and is estimated at $30-50 \%$. Assessing adherence is an important part of medicines optimisation (NICE, 2015b). A medication that is prescribed or even issued regularly cannot be presumed to be taken correctly, to maximal effect and without side-effects. Clinicians should endeavour to check how a medication is being taken by the patient and the issue history on the clinical system can help to trigger and support these discussions. The NICE Medicines Optimisation Guidance recommends the use of patient decision aids to assist with shared decision making around prescribing. NHS patient decision aids are available as applications to download to mobile devices or at http://patient.info/decision-aids

Examples of errors include: 
$>$ Patient collecting Clenil $^{\circledR}$ regularly but not collecting separate long acting beta agonist (LABA) inhaler. A medication review had been coded recently but non-adherence had not been addressed.

> Asthma inhalers for a child being issued at a higher than expected frequency (inhaled corticosteroid and salbutamol). The parent requested further supply due to running out. Further supply was issued without further questioning as to the child's asthma control.

$>$ Fluoxetine being collected intermittently at a frequency where daily dosing would not be possible and a medication review coded without discussing antidepressant usage.

\section{Right documentation.}

The clinical record should document the necessary clinical information and checks to support prescribing. This is important even if the indication can be deduced from the item prescribed.

Examples of errors include:

Strong opioids prescribed for the first time during a consultation with no entry in the records indicating the reason for the prescription.

$>$ Combined oral contraceptive prescribed without recording information on smoking status, weight etc.

$>$ Potent topical steroid started without a clinical indication.

$>$ Diazepam prescribed for the first time to a patient who was seen with an interpreter present. The reason for prescribing was not in the consultation notes.

The prescribing of some medications should trigger consideration of a second medication to be given concurrently e.g. the prescribing of an NSAID to a patient over 65 years would require consideration of gastroprotection. If the item is considered but not required, or declined by the patient, then this should be documented so that other clinicians who access the record are also aware so that it can inform their decisions. An example of this type of error is the prescribing of a strong opioid without documented consideration of co-prescribing a laxative.

\section{Right prescribing experience.}


GP AiTs should work with GP trainers to ensure that adequate prescribing experience is gained in all the areas that will be needed post qualification. In the 100 prescription study, five categories of medication accounted for almost $50 \%$ of prescribing with almost two-thirds of prescribing for new acute items. This pattern of prescribing would seem to be different than national prescribing figures for GPs, with a lesser propensity for the GP AiTs to be prescribing medication for long term conditions (Prescribing and Medicines Team Health and Social Care Information Centre, 2017). If on reviewing their own prescribing, a GP AiT were to find similar results, they may wish to consider how they could expand their prescribing experience within primary care. GP trainers who reflected on these results in relation to their own GP AiTs provided the following quotes:

$>$ "I guess one of the things that it revealed to me, which didn't come as a surprise, was the fact that most of his prescribing is in acute prescribing, but I think that's generally the case for our registrars..."

$>$ "I think one of the real things here is the fact that the registrars see patients presenting for acute problems on the day, and I might consider again how I might get the appropriate case mix, because I think it's a reflection of the fact that they're not seeing the same patient case-mix as I am..."

$>$ "When a lot of (his/her) patients who are started on what would be long-term medication... that really confirmed the fact that (he/she) wasn't very good at putting (medicines) on repeats..."

\section{Summary}

Prescribing in general practice is a complicated and integral part of the role of a GP. It is therefore important to ensure GP AiTs feel confident in prescribing and develop good prescribing habits during their training so that there is a durable reduction in the risk of prescribing errors in the long term. 
There are many examples of clinical pharmacists working with GP practices to provide training support and facilitate safer prescribing. There are plans to evaluate the impact of the Clinical Pharmacists in General Practice Programme.

It is hoped that GP AiTs and GP trainers can use the information provided in this article to review their own prescribing and tailor future training. However, when considering safer prescribing, the top tips provided here should be considered alongside other available prescribing guides and on-line learning resources available.

\section{Insert box 1}

\section{Key points}

- Developing good practice prescribing skills as a GP AiT could lead to life-long habits that will defend against prescribing error

- Pharmacists can work effectively as part of the practice team to enhance prescribing safety and support GP AiTs

- Analysing a sample of prescriptions is a useful educational tool for prescribers to review and improve their prescribing habits

- Prescribing guidelines and formularies should be readily accessible and up to date

- Adherence to medication should always be assessed when changing or reviewing medication 


\section{References and further information}

- Avery T, 10 Top tips for GPs - Strategies for safer prescribing. (2011) Liverpool: National Prescribing Centre. Retrieved from https://bibliosjd.files.wordpress.com/2010/12/prescription.pdf

- Avery, A. J., Barber, N., Ghaleb, M., Franklin, B.D., Armstrong, S., Crowe, S., . . ,Talabi, O. (2012).Investigating the prevalence and causes of prescribing errors in general practice: The PRACtICe study. Retrieved from www.gmc-uk.org/about/research/12996.asp

- Avery T, Gookey G, Spencer R, Knox R, Marsden K, Salema N. (2013a) Selecting the right drug. InnovAIT, 6 (8): 478-487. doi: 10.1177/1755738013491400

- Avery T, Gookey G, Spencer R, Knox R, Marsden K, Salema N. (2013b) Avoiding hazardous prescribing. InnovAIT, 6 (8): 488-496. doi: 10.1177/1755738013494370

- Avery T, Gookey G, Spencer R, Knox R, Marsden K, Salema N. (2013c) Selecting the right dose. InnovAIT, 6 (8): 497-505. doi:10.1177/1755738013494365

- Avery T, Gookey G, Spencer R, Knox R, Marsden K, Salema N. (2013d) Providing the right dose instructions. InnovAIT, 6 (8): 506-514. doi:10.1177/1755738013494367

- Avery T, Gookey G, Spencer R, Knox R, Marsden K, Salema N.(2013e) Providing the right medication monitoring. InnovAIT, 6 (8): 515-523. doi:10.1177/1755738013494368

- Avery T, Gookey G, Spencer R, Knox R, Marsden K, Salema N. (2013) Undertaking effective medication reviews. InnovAIT, 6 (8): 524-533. doi:10.1177/1755738013494369

- BMJ Group \& Pharmaceutical Press. (2017a). British national formulary for children. Retrieved from www.medicinescomplete.com/mc/bnfc/current/

- BMJ Group \& Pharmaceutical Press. (2017b). British national formulary for children. Retrieved from www.medicinescomplete.com/mc/bnf/current/

- Kessels, R. P. C. (2003). Patients' memory for medical information. Journal of the Royal Society of Medicine, 96(5), 219-222. doi: 10.1258/jrsm.96.5.219

- MHRA. (2011). Citalopram and escitalopram: QT interval prolongation - new maximum daily dose restrictions (including in elderly patients), contra-indications and warnings. Retrieved from www.gov.uk/drug-safety-update/citalopram-and-escitalopram-qtinterval-prolongation

- NHS England. (2014). The NHS five year forward view. Retrieved from www.england.nhs.uk/wp-content/uploads/2014/10/5yfv-web.pdf

- NHS England. (2016). The General Practice Forward View. Retrieved from www.england.nhs.uk/gp/gpfv/

- NHS England. (2017). Next steps on the NHS five year forward view. Retrieved from www.england.nhs.uk/wp-content/uploads/2017/03/NEXT-STEPS-ON-THE-NHS-FIVEYEAR-FORWARD-VIEW.pdf

- NICE. (2015a). Antimicrobial stewardship: systems and processes for effective antimicrobial medicine use. Retrieved from www.nice.org.uk/guidance/ng15 
- NICE. (2015b). Medicines optimisation: the safe and effective use of medicines to enable the best possible outcomes. Retrieved from www.nice.org.uk/guidance/ng5

- Prescribing and Medicines Team Health and Social Care Information Centre. Prescriptions dispensed in the community (England 2005-2015). (2016). Health and Social Care Information system, London. Retrieved from http://content.digital.nhs.uk/catalogue/PUB20664/pres-disp-com-eng-2005-15-rep.pdf

- RCGP. Professional Module 2.04: Enhancing Professional Knowledge. Retrieved from www.rcgp.org.uk/training-exams/gp-curriculum-overview/online-curriculum/working-insystems-of-care/2-04-enhancing-professional-knowledge.aspx

- Royal College of Physicians. (2016). National clinical guideline for stroke. Fifth edition. Retrieved from www.strokeaudit.org/Guideline/Full-Guideline.aspx

\section{Box 1. Examples of learning resources available to support safer prescribing}

- The RCGP e-learning site contains a course entitled "Prescribing in general practice". This course contains five modules that cover various aspects of safe prescribing:

- Appropriate drug selection

- Avoiding prescribing errors

- Choosing the right dose

- Right dose

- Effective medication reviews

- 10 Top tips for GPs - Strategies for safer prescribing. Produced by the National Prescribing Centre (Avery, 2011).

- The Special edition of InnovAiT which highlighted lessons learned from the PRACtICe Study (Avery, Gookey, Spencer, Knox, Marsden \& Salema. (2013a-e). 